\title{
Association between dietary patterns and anaemia in adults from Jiangsu Province in Eastern China
}

\author{
Zumin Shi ${ }^{1,2}$, Xiaoshu $\mathrm{Hu}^{1}$, Baojun Yuan ${ }^{1}$, Xiaoqun Pan $^{1}$, Yue Dai ${ }^{1}$ and Gerd Holmboe-Ottesen ${ }^{2}$ \\ ${ }^{1}$ Jiangsu Provincial Center for Disease Control and Prevention, Nanjing, China \\ ${ }^{2}$ Section of Preventive Medicine and Epidemiology, Institute of General Practice and Community Medicine, University of Oslo, \\ PO Box 1130, N-0318 Oslo, Norway
}

(Received 20 October 2005 - Revised 10 February 2006 - Accepted 21 February 2006)

\begin{abstract}
The objective of the present study was to investigate the association between food patterns and anaemia among Chinese adults. It was a crosssectional household survey undertaken in 2002. The sample contained 2849 men and women aged 20 years and above, and had a response rate of $89.0 \%$. Factor analysis was used to identify food patterns based on a food-frequency questionnaire. Logistic regression was used to relate food patterns to anaemia. A four-factor solution explained $30.5 \%$ of the total variance. After adjusting for socio-demographic factors and four distinct food patterns, the 'traditional' (rice, vegetable, wheat flour), 'sweet tooth' (drinks, cake) and 'healthy' (whole grains, fruits, vegetables) patterns were independently associated with anaemia. 'Traditional' and 'sweet tooth' patterns were positively associated with anaemia, whereas the association with 'healthy' food pattern was negative. No association was observed between the 'macho' pattern (meat and alcohol) and anaemia. Compared with the lowest quartile (Q1) of the 'traditional' pattern, the highest quartile (Q4) had a higher risk of anaemia (men: odds ratio (OR) 2.60, $95 \%$ CI 1.38, 4.88; women: OR 3.40, $95 \%$ CI 2.14, 5.39). For the 'sweet tooth' pattern, compared with the lowest quartile (Q1), the OR of the highest quartile was $2.34(95 \%$ CI $1.47,3.73)$ for men and $2.02(95 \%$ CI $1.31,3.13)$ for women. The fourth quartile of healthy food was associated with a lower risk of anaemia (men: OR $0.50,95 \%$ CI $0.31,0.79$; women: OR 0.51 , $95 \%$ CI $0.34,0 \cdot 75$ ). Women in the north had a higher risk of anaemia (OR 2.49, $95 \%$ CI 1.80, 3.43). Food patterns were associated with anaemia in this area with a high prevalence of anaemia.
\end{abstract}

Food pattern: Factor analysis: Anaemia: China

Iron-deficiency anaemia is a health problem mainly affecting developing countries despite the fact that its causes are well known (ACC/SCN, 2000). The prevalence of anaemia is $15.2 \%$ in China ( $\mathrm{Li}$ et al. 2005). No appropriate preventive measure has yet been taken against this public health problem. The only measure tried has been Fe-fortified soya sauce, which was supplied on the market in some areas in China as recently as 2004 (Chen et al. 2005). Thus, the prevention of anaemia is still a challenge.

Several factors are found to be associated with anaemia, but the leading causes in developing countries are iron deficiency, infectious diseases and other nutritional deficiencies that influence $\mathrm{Hb}$ metabolism (World Health Organization, 2001). Iron-deficiency anaemia is mainly associated with the amount of $\mathrm{Fe}$ in the diet, chemical forms of $\mathrm{Fe}$, enhancers and inhibitors of Fe bioavailability, and individual Fe status (Harvey et al. 2000; Heath \& Fairweather-Tait, 2002; Boccio \& Iyengar, 2003). Vitamin A deficiency, folate deficiency and infection are found to be associated with iron-deficiency anaemia (Semba \& Bloem, 2002). The present knowledge about the link between dietary factors and risk of iron-deficiency anaemia is focused on individual nutrients or food items. In real life, people eat a variety of different foods, which give several nutrients a chance to interact. In addition, foods contain both inhibitors and enhancers of Fe bioavailability, which may interact.

There is a growing interest in the relation between dietary patterns and disease, for example cardiovascular disease, type 2 diabetes and colon cancer (Jacques \& Tucker, 2001; Hu, 2002). Factor analysis, cluster analysis and the use of dietary indices are the main methods of determining dietary patterns ( $\mathrm{Hu}, 2002)$. In recent years, several reviews on the application of factor analysis in nutritional epidemiology have been published (Togo et al. 2001; Kant, 2004; Newby \& Tucker, 2004). Many of these published studies focus on the association between dietary pattern and cancer, diabetes and obesity. To better understand the association between diet and anaemia, it is important to describe the association between food patterns and anaemia. No study has been reported that describes this relationship by using factor analysis.

The objective of the present study is to investigate dietary patterns in relation to risk of anaemia using household survey data from Jiangsu, China. 


\section{Materials and methods}

\section{Sample}

In 2002, China launched a national study in nutrition and health under the approval of the Chinese Ministry of Health. A multistage cluster sampling method was used to select the participants. The data presented in this article are based on a subsample from Jiangsu province, one of the economically booming areas in China, with a population of 73.6 million. The rural sample was selected from six counties (Jiangyin, Taicang, Suining, Jurong, Sihong, Haimen). From each of the six counties, three towns were randomly selected. The urban sample was selected from two prefecture capital cities (Nanjing, Xuzhou). Three streets were randomly selected from each prefecture.

The six counties and two prefectures represented a geographically and economically diverse population with gross domestic product ranging from 3221 Yuan/capita/year (US\$403) to 35169 Yuan/capita/year (US\$4396; mean US\$1993, SD 1510; Jiangsu Bureau of Statistics, 2002). Nanjing, Jurong, Taicang and Jiangyin are in the South. The south has higher gross domestic product than the north (24702 v. 7183 Yuan).

In each town/street, two villages/neighbourhoods were further randomly selected. In each village/neighbourhood, thirty households were randomly selected. All members in the households were invited to take part in the study. Written consent was obtained from all the participants. In the study presented here, we only analysed data for adults aged 20 years and above. The total sample included 1308 men and 1541 women, of whom 711 came from the urban area. The response rate was $89.0 \%$.

\section{Measurements}

Anaemia. Hb level was measured by the cyanmethaemoglobin method (Dallman, 1984). Blood sample collection, transportation, storage and testing in standardised laboratories in each province were performed according to protocol developed by the Chinese Center for Disease Control and Prevention. All reagents and blood-sampling tools were provided by, and the quality of examination was supervised by professionals from, the Center. Anaemia was defined as a $\mathrm{Hb}$ level of below $13 \mathrm{~g} / \mathrm{dl}$ for men and $12 \mathrm{~g} / \mathrm{dl}$ for women (World Health Organization, 2001).

Dietary data, health habits and socio-demographic information. Participants were interviewed in their homes by trained health workers from the local Center for Disease Control and Prevention. The interviews took approximately $2 \mathrm{~h}$ to complete and included questions on diet, socio-demographic information, health habits such as cigarette smoking and physical activity, and other lifestyle factors. Diet over the previous year was investigated using a series of detailed questions regarding the usual frequency and quantity of intake of thirty-three foods and beverages. This was further merged into twenty-five food items in the analysis because of the low intake of some food items. Portion size for each food was established by reference to food models. Subjects were asked to recall the frequency of consumption of individual food items (number of times per day, per week, per month, per year) and the estimated portion size, using local weight units (1 liang $=50 \mathrm{~g}$ ) or natural units (cups). Intakes of foods were converted into g/week and were used in the further analysis. Vitamin and $\mathrm{Fe}$ supplements were included in the questionnaire. Because these are very seldom used in the area, they were not included in the analysis.

The food-frequency questionnaire has been validated (Zhao et al. 2002). According to the validation study, the food-frequency questionnaire is a useful method for collecting individual food consumption information in a face-to-face interview, but is not useful in self-administered surveys owing to the current educational level of the majority in the Chinese population.

Dietary patterns were identified by factor analysis, using standard principal component analysis method (Kim \& Mueller, 1978). Factors were rotated with an orthogonal (varimax) rotation to improve interpretability and minimise the correlation between the factors. The number of factors retained from each food classification method was determined by factor interpretability and the amount of variance explained by each factor. Labelling of the factors was primarily descriptive and based on our interpretation of the pattern structures.

Participants were assigned pattern-specific factor scores. Scores for each pattern were calculated as the sum of the products of the factor loading coefficient and the standardised weekly intake of each food associated with that pattern. Only foods with factor loadings of more than 0.20 and less than -0.20 were included in calculation of pattern scores because these items represent the foods most strongly related to the identified factor.

Socio-economic status (SES) was assessed by the question 'What was your family's income per person in 2001?' The response categories for the question were less than 800 , 800-1999, 2000-4999, 5000-9999, 10 000-19 999 and more than 20000 Yuan. SES was constructed from income, 'low' being less than 1999 Yuan, 'medium' being 2000-4999 Yuan and 'high' being more than 5000 Yuan.

\section{Statistical analyses}

Factor scores were divided into quartiles. The scores (intakes) increased from quartile 1 (Q1) to quartile 4 (Q4). The chi-squared test was used to compare the difference between categorical variables. Logistic regression was used to determine the association between food pattern and anaemia adjusted for socio-demographic factors including residence, age, socioeconomic status, region and education. Multivariate linear regression was used to describe the associations between $\mathrm{Hb}$ and factor scores. All the analyses were performed using SPSS 11.0 (SPSS Inc., Chicago, IL, USA).

\section{Results}

Table 1 shows the sample characteristics. The prevalence of anaemia was $18.3 \%$ and $31.5 \%$ for men and women, respectively. The south had a higher prevalence of anaemia than the north $(29.0 \%$ v. $21.5 \%)$.

Four food patterns were obtained by factor analysis. Factor loadings (equivalent to simple correlations between the food items and the dietary patterns) for the four food patterns and the name assigned to each pattern are presented in Table 2. The 'traditional' pattern (factor 1) loaded heavily on rice 
Table 1. Sample characteristics

\begin{tabular}{|c|c|c|c|c|c|}
\hline & & \multicolumn{2}{|c|}{ Male } & \multicolumn{2}{|c|}{ Female } \\
\hline & & $n$ & $\%$ & $n$ & $\%$ \\
\hline \multicolumn{2}{|l|}{ Total } & 1308 & $45 \cdot 9$ & 1541 & $54 \cdot 1$ \\
\hline \multirow[t]{5}{*}{ Age group (years) } & $20-30$ & 164 & $12 \cdot 5$ & 201 & $13 \cdot 0$ \\
\hline & $31-40$ & 314 & $24 \cdot 0$ & 399 & $25 \cdot 9$ \\
\hline & $41-50$ & 319 & $24 \cdot 4$ & 379 & $24 \cdot 6$ \\
\hline & $51-60$ & 255 & $19 \cdot 5$ & 283 & $18 \cdot 4$ \\
\hline & $\geq 61$ & 256 & $19 \cdot 6$ & 279 & $18 \cdot 1$ \\
\hline \multirow[t]{2}{*}{ Residence } & Urban & 330 & $25 \cdot 2$ & 381 & $24 \cdot 7$ \\
\hline & Rural & 978 & $74 \cdot 8$ & 1160 & $75 \cdot 3$ \\
\hline \multirow[t]{2}{*}{ Region } & South & 702 & $53 \cdot 7$ & 802 & $52 \cdot 0$ \\
\hline & North & 606 & $46 \cdot 3$ & 739 & $48 \cdot 0$ \\
\hline \multirow[t]{4}{*}{ Education } & Primary & 471 & $36 \cdot 0$ & 886 & 57.5 \\
\hline & Junior school & 569 & 43.5 & 464 & $30 \cdot 1$ \\
\hline & High school & 213 & $16 \cdot 3$ & 166 & $10 \cdot 8$ \\
\hline & University & 55 & $4 \cdot 2$ & 24 & 1.6 \\
\hline \multirow[t]{3}{*}{ Socio-economic status } & Low & 419 & $32 \cdot 3$ & 495 & $32 \cdot 4$ \\
\hline & Medium & 413 & 31.9 & 500 & $32 \cdot 7$ \\
\hline & High & 464 & $35 \cdot 8$ & 532 & 34.8 \\
\hline \multicolumn{2}{|l|}{ Anaemia } & 240 & $18 \cdot 3$ & 485 & 31.5 \\
\hline
\end{tabular}

and fresh vegetable and inversely on wheat flour. Factor 2 ('macho') was characterised by various kinds of animal foods and alcohol, i.e. foods commonly eaten by men. Factor 3 ('sweet tooth') contained cake, milk, yoghurt and drinks. Factor 4 ('healthy') included whole grains, fruit, root vegetables and fresh and pickled vegetables. The four factors explained $30.5 \%$ of the variance in intake $(10.6 \%, 8.6 \%$, $5.9 \%$ and $5.4 \%$ for factors 1 to 4 , respectively).

Compared with the lowest quartile (Q1) of the 'traditional' pattern, the highest quartile (Q4) had a higher mean age (Table 3). For the other three food patterns, the highest quartile groups had a lower mean age. The high-SES group had the highest percentage of 'traditional', 'macho' and 'sweet tooth' food in the fourth quartile, whereas the low-SES group had the highest proportion in both the highest quartile of 'healthy' food and the lowest quartile of 'traditional' and 'sweet tooth' foods. Urban residents had a higher proportion in the highest quartile of 'macho' and 'sweet tooth' food compared with the rural residents $(39.8 \%$ and $49.6 \%$ v. $20.1 \%$ and $16.8 \%$, respectively). Men had a higher intake of 'macho' food, whereas women had a higher intake of 'sweet tooth' (for both food patterns, $P<0 \cdot 01$ ). No gender difference was observed in the 'healthy' pattern. The regional differences in food pattern were large. People from the north had a lower proportion of the 'traditional' pattern and a higher proportion of the 'healthy' pattern.

The mean intake of animal food was $59.9 \mathrm{~g} / \mathrm{d}$ (data not shown). The highest animal food intake $(119 \mathrm{~g} / \mathrm{d})$ was found in fourth quartile of the 'macho' pattern. In all the other groups, the mean intake of animal food was below $90 \mathrm{~g} / \mathrm{d}$. In the fourth quartile of the 'healthy' pattern, the mean intakes of fresh vegetables and fruits were 310 and $114 \mathrm{~g} / \mathrm{d}$, respectively (Table 3 ).

Table 4 shows the prevalence of anaemia across the quartiles of the four food factors. Factors 1 and 3 were positively associated with the prevalence of anaemia. Men from first quartile of the 'traditional' pattern had the lowest prevalence of anaemia ( $7.7 \%$ ), compared with $24.8 \%$ in the fourth quartile group. The prevalence of anaemia in fourth quartile of the 'healthy' food group was half that in the first quartile, for both men and women. A trend of negative association between 'macho' food and anaemia was found among men, although it was not statistically significant. Men from the first quartile of the 'macho' food group had the highest prevalence of anaemia (23.6\%), compared with $16.9 \%$ in the fourth quartile group. There was, however, no such association among women. The highest prevalence of anaemia (42.4\%) was found in the group of women in first quartile of the 'healthy' pattern.

Results from logistic regression show that, after adjusting for socio-demographic factors, factor 1 ('traditional'), factor 3 ('sweet tooth') and factor 4 ('healthy') were independently associated with anaemia in both genders (Table 5). Factors 1 and 3 were positively associated with anaemia. Compared with the lowest quartile (Q1) of factor 1, the highest quartile (Q4) had a higher risk of anaemia (men: odds ratio (OR) 2.60, $95 \%$ CI 1.38, 4.88; women: OR 3.40, $95 \%$ CI 2.14, 5.39).

Table 2. Factor loadings* for four food patterns among adults in Jiangsu, China ( $n$ 2849)

\begin{tabular}{|c|c|c|c|c|c|c|c|}
\hline \multicolumn{2}{|c|}{ Factor 1: 'Traditional' } & \multicolumn{2}{|c|}{ Factor 2: 'Macho’ } & \multicolumn{2}{|c|}{ Factor 3: 'Sweet tooth' } & \multicolumn{2}{|c|}{ Factor 4: ‘Healthy’ } \\
\hline $\begin{array}{l}\text { Food or } \\
\text { food group }\end{array}$ & $\begin{array}{l}\text { Factor } \\
\text { loading }\end{array}$ & $\begin{array}{l}\text { Food or } \\
\text { food group }\end{array}$ & $\begin{array}{l}\text { Factor } \\
\text { loading }\end{array}$ & $\begin{array}{l}\text { Food or } \\
\text { food group }\end{array}$ & $\begin{array}{l}\text { Factor } \\
\text { loading }\end{array}$ & $\begin{array}{l}\text { Food or } \\
\text { food group }\end{array}$ & $\begin{array}{l}\text { Factor } \\
\text { loading }\end{array}$ \\
\hline Rice & 0.81 & Poultry & 0.56 & Cake & 0.60 & Whole grains & 0.54 \\
\hline Fresh vegetables & 0.57 & Beer & 0.53 & Juice & 0.58 & Fruits & 0.49 \\
\hline Pork & 0.37 & Beef, lamb & 0.46 & Beverage & 0.48 & Pickled vegetables & 0.46 \\
\hline Fish & 0.21 & Deep-fried products & 0.45 & Milk & 0.48 & Tofu & 0.44 \\
\hline Root vegetables & -0.32 & Pork & 0.44 & Yoghurt & 0.44 & Fresh vegetables & 0.37 \\
\hline \multirow{7}{*}{ Wheat flour† } & -0.78 & Liver & 0.43 & Beef, lamb & 0.30 & Root vegetables & 0.34 \\
\hline & & Alcohol & 0.43 & Nut & 0.26 & Milk & 0.31 \\
\hline & & Eggs & 0.38 & Poultry & 0.25 & Eggs & 0.30 \\
\hline & & Fish & 0.26 & Fruits & 0.23 & Fish & 0.24 \\
\hline & & Nuts & 0.23 & Pickled vegetables & -0.20 & Wheat flour & 0.23 \\
\hline & & Fruits & 0.22 & Alcohol & -0.27 & Milk powder & 0.22 \\
\hline & & Tofu & 0.22 & & & Beer & -0.21 \\
\hline
\end{tabular}

* Factor loadings are equivalent to a simple correlation between the food items and the factor. Higher loadings (absolute value) indicate that the food shares more variance with that factor. The sign of the loading determines the direction of the relationship of each food to the factor. Food groups with absolute values of less than 0.20 are excluded from the table for simplicity. Only one food item (cheese) is missing owing to low factor loading.

† Wheat flour includes noodles and steamed dumplings.

$\ddagger$ Beverage includes soft drinks, coffee and tea. 
Table 3. Sample characteristics for the lowest (Q1) and highest (Q4) quartiles of four food patterns among adults in Jiangsu, China ( $n$ 2849)

\begin{tabular}{|c|c|c|c|c|c|c|c|c|c|c|c|c|c|c|c|c|}
\hline & \multicolumn{4}{|c|}{ Factor 1: 'Traditional' $\dagger$} & \multicolumn{4}{|c|}{ Factor 2: 'Macho'† } & \multicolumn{4}{|c|}{ Factor 3: 'Sweet tooth’† } & \multicolumn{4}{|c|}{ Factor 4: 'Healthy'† } \\
\hline & \multicolumn{2}{|c|}{ Q1 } & \multicolumn{2}{|c|}{ Q4 } & \multicolumn{2}{|c|}{ Q1 } & \multicolumn{2}{|c|}{ Q4 } & \multicolumn{2}{|c|}{ Q1 } & \multicolumn{2}{|c|}{ Q4 } & \multicolumn{2}{|c|}{ Q1 } & \multicolumn{2}{|c|}{ Q4 } \\
\hline Mean & \multicolumn{2}{|c|}{$43 \cdot 6$} & \multicolumn{2}{|c|}{$47 \cdot 8$} & \multicolumn{2}{|c|}{$51 \cdot 5$} & \multicolumn{2}{|c|}{$43 \cdot 1$} & \multicolumn{2}{|c|}{$46 \cdot 8$} & \multicolumn{2}{|c|}{$44 \cdot 3$} & \multicolumn{2}{|c|}{$49 \cdot 0$} & \multicolumn{2}{|c|}{46.5} \\
\hline SD & \multicolumn{2}{|c|}{0.5} & \multicolumn{2}{|c|}{0.5} & & 5 & & & & .5 & 0 & 5 & & .5 & & 5 \\
\hline $\mathrm{Hb}$ & & & & & & & & & & & & & & & & \\
\hline Mean & 14 & & 13 & & 13 & .4 & 13 & & 13 & & 13 & & 13 & & 13 & \\
\hline Gender & & & & & & & & & & & & & & & & \\
\hline Male (n 1308) & 26 & & 29 & & 17 & & 35 & & 34 & & 22 & & 26 & & 24 & $5^{\star}$ \\
\hline Female $(n$ 1541) & 24 & & 21 & & 31 & & 15 & & 16 & & 27 & & 23 & & 25 & \\
\hline Socio-economic status & & & & & & & & & & & & & & & & \\
\hline Low $(n 914)$ & 56 & & & $\cdot 2$ & 36 & & 17 & & 34 & & 11 & & 18 & & 30 & \\
\hline Medium (n913) & 16 & & 32 & & 23 & & 27 & & 27 & & 21 & & 25 & & 21 & \\
\hline High (n 996) & & $\cdot 2$ & 33 & & 15 & & 29 & & 14 & & 41 & & 30 & $\cdot 1$ & 23 & \\
\hline Education & & & & & & & & & & & & & & & & \\
\hline Junior school (n 1033) & 24 & & 25 & & 15 & & 32 & & 26 & & 26 & & 22 & .9 & 24 & \\
\hline High school (n 379) & 17 & & 27 & & 16 & & 35 & & 17 & & 46 & & 19 & .5 & 33 & \\
\hline University (n 79) & 16 & & 31 & & 15 & .2 & 43 & & 3 & 8 & 70 & & 10 & $\cdot 1$ & 44 & \\
\hline Residence & & & & & & & & & & & & & & & & \\
\hline Urban (n711) & 22 & & 18 & & 13 & 8 & 39 & & 14 & & 49 & & 10 & $\cdot 8$ & 38 & \\
\hline Rural (n 2138) & 25 & & 27 & & 28 & .7 & 20 & & 28 & & 16 & & 29 & .7 & 20 & \\
\hline Region & & & & & & & & & & & & & & & & \\
\hline South (n 1504) & & $\cdot 8$ & 39 & $\cdot 0$ & 18 & 6 & 26 & & 16 & & 32 & & 34 & 6 & 19 & \\
\hline North (n 1345) & 52 & & & 3 & 32 & $\cdot 1$ & 23 & & 34 & & 16 & & 14 & $\cdot 1$ & 30 & \\
\hline & Mean & SD & Mean & SD & Mean & SD & Mean & SD & Mean & SD & Mean & SD & Mean & SD & Mean & SD \\
\hline Food intake $(\mathrm{g} / \mathrm{d})$ & & & & & & & & & & & & & & & & \\
\hline Animal foods & 33 & 40 & 87 & 78 & 18 & 20 & 119 & 80 & 52 & 64 & 89 & 70 & 58 & 60 & 69 & 69 \\
\hline Rice & 87 & 78 & 467 & 143 & 273 & 190 & $280^{*}$ & 165 & 294 & 207 & 263 & 134 & 342 & 170 & 231 & 162 \\
\hline Wheat flour & 377 & 176 & 22 & 34 & 156 & 205 & $144^{*}$ & 173 & 220 & 233 & 84 & 118 & 64 & 129 & 213 & 214 \\
\hline Whole grains & 16 & 31 & 4 & 18 & 12 & 33 & 8 & 14 & 15 & 31 & 10 & 25 & 1 & 3 & 24 & 38 \\
\hline Fresh vegetables & 154 & 105 & 375 & 205 & 263 & 164 & $251^{*}$ & 176 & 317 & 226 & 243 & 133 & 193 & 100 & 310 & 233 \\
\hline
\end{tabular}

†The 'traditional' pattern includes rice and vegetables; the 'macho' pattern includes animal foods and alcohol; the 'sweet tooth' pattern includes cake, drinks, milk and yoghurt; the 'healthy' pattern includes mainly whole grains, fruits, vegetables, tofu and tubers. $\ddagger$ Adjusted for age and sex.

${ }^{*} P>0.05$.

For factor 3, compared with the lowest quartile (Q1), the OR of highest quartile was 2.34 for men and 2.02 for women. Factor 4 ('healthy') was inversely associated with anaemia. The fourth quartile of healthy food was associated with a lower risk of

Table 4. Unadjusted prevalence (\%) of anaemia across quartiles (Q1-Q4) of four food factors in Jiangsu, China ( $n$ 2849)*

\begin{tabular}{lrrrrr}
\hline & Q1 & Q2 & Q3 & Q4 & \multicolumn{1}{c}{$P$} \\
\hline Men & & & & & \\
$\quad$ Factor 1: 'Traditional' & 7.7 & 17.7 & 22.6 & 24.8 & $<0.001$ \\
$\quad$ Factor 2: 'Macho' & 23.6 & 19.8 & 15.5 & 16.9 & 0.074 \\
$\quad$ Factor 3: 'Sweet tooth' & 12.6 & 21.2 & 21.5 & 21.5 & 0.002 \\
$\quad$ Factor 4: 'Healthy' & 28.5 & 20.1 & 11.0 & 12.5 & $<0.001$ \\
Women & & & & & \\
$\quad$ Factor 1: 'Traditional' & 18.1 & 34.5 & 38.3 & 33.8 & $<0.001$ \\
Factor 2: 'Macho' & 30.5 & 30.3 & 34.3 & 30.9 & 0.583 \\
$\quad$ Factor 3: 'Sweet tooth' & 21.5 & 26.3 & 38.9 & 34.1 & $<0.001$ \\
Factor 4: 'Healthy' & 42.4 & 29.7 & 30.8 & 23.5 & $<0.001$ \\
\hline
\end{tabular}

*The 'traditional' pattern includes rice and vegetables; the 'macho' pattern includes animal foods and alcohol; the 'sweet tooth' pattern includes cake, drinks, milk and yoghurt; the 'healthy' pattern includes mainly whole grains, fruits, vegetables, tofu and tubers. anaemia (men: OR 0.50, $95 \%$ CI 0.31, 0.79; women: OR 0.51, $95 \%$ CI $0.34,0.75)$. No association was observed between 'macho' food and anaemia. Women from the north had a 2.49 times higher risk of being anaemic than women from the south. Use of a multivariate linear regression model including the four factors and socio-demographic factors showed similar results. The associations between $\mathrm{Hb}$ and the factor scores were significant except for factor 2 . The regression coefficients were $-2 \cdot 81,-0 \cdot 06,-0.80$ and $2 \cdot 17$ for factors 1 to 4 , respectively (data not shown).

\section{Discussion}

In a cross-sectional study, we investigated the association between dietary patterns and anaemia among adults in Jiangsu, China. Of the four food patterns we obtained, the 'traditional' and 'sweet tooth' food patterns were positively associated with anaemia, whereas the 'healthy food' pattern was inversely associated with anaemia.

The present study had its strengths and limitations. It included an economically and geographically diverse population with a high prevalence of anaemia. This made it possible to study the association between food patterns 
Table 5. Odds ratio* from multivariate logistic regression assessing associations between food factors and anaemia in Jiangsu, China ( $n$ 2849)

\begin{tabular}{|c|c|c|c|c|c|c|}
\hline & \multicolumn{3}{|c|}{ Men } & \multicolumn{3}{|c|}{ Women } \\
\hline & Odds ratio & $95 \% \mathrm{Cl}$ & $P$ & Odds ratio & $95 \% \mathrm{Cl}$ & $P$ \\
\hline \multicolumn{7}{|l|}{ Residence } \\
\hline Urban & 1 & & & 1 & & \\
\hline Rural & $1 \cdot 23$ & $0.80,1.90$ & 0.345 & 1.03 & $0.75,1.41$ & 0.866 \\
\hline \multicolumn{7}{|c|}{ Age group (years) } \\
\hline $20-30$ & 1 & & & 1 & & \\
\hline $31-40$ & 1.06 & $0.54,2.07$ & 0.864 & 0.46 & $0.31,0.68$ & $<0.001$ \\
\hline $41-50$ & $2 \cdot 11$ & $1.13,3.95$ & 0.019 & 0.71 & $0.47,1.07$ & 0.106 \\
\hline $51-60$ & 2.09 & $1.09,4.00$ & 0.026 & 0.62 & $0.40,0.98$ & 0.039 \\
\hline$\geq 61$ & 3.34 & $1 \cdot 71,6 \cdot 50$ & $<0.001$ & 0.78 & $0.48,1.24$ & 0.293 \\
\hline \multicolumn{7}{|c|}{ Socio-economic status } \\
\hline Low & 1 & & & 1 & & \\
\hline Medium & 1.07 & $0.68,1.67$ & 0.770 & $2 \cdot 08$ & $1 \cdot 49,2 \cdot 89$ & $<0.001$ \\
\hline High & 0.81 & $0.50,1.31$ & 0.393 & 1.95 & $1 \cdot 35,2 \cdot 82$ & $<0.001$ \\
\hline \multicolumn{7}{|l|}{ Education } \\
\hline Primary & 1 & & & 1 & & \\
\hline Junior & 0.87 & $0.60,1.26$ & 0.450 & 0.90 & $0.65,1.23$ & 0.500 \\
\hline Senior & 0.72 & $0.42,1.23$ & 0.226 & 0.85 & $0.55,1.30$ & 0.444 \\
\hline University & 0.53 & $0.20,1.40$ & 0.201 & 0.33 & $0.10,1.09$ & 0.068 \\
\hline \multicolumn{7}{|c|}{ 'Traditional' pattern† } \\
\hline Q1 & 1 & & & 1 & & \\
\hline Q2 & 1.90 & $1 \cdot 06,3 \cdot 38$ & 0.030 & $2 \cdot 27$ & $1 \cdot 55,3 \cdot 33$ & $<0.001$ \\
\hline Q3 & 1.87 & $0.99,3.55$ & 0.054 & $3 \cdot 18$ & $2.06,4.90$ & $<0.001$ \\
\hline Q4 & $2 \cdot 60$ & $1 \cdot 38,4.88$ & 0.003 & 3.40 & $2 \cdot 14,5 \cdot 39$ & $<0.001$ \\
\hline \multicolumn{7}{|c|}{ 'Macho' pattern† } \\
\hline Q1 & 1.00 & $0.00,0.00$ & 0.212 & 1 & & \\
\hline Q2 & 0.83 & $0.52,1.33$ & 0.436 & 0.85 & $0.62,1.16$ & 0.296 \\
\hline Q3 & 0.64 & $0.40,1.04$ & 0.074 & $1 \cdot 10$ & $0.79,1.53$ & 0.567 \\
\hline Q4 & 0.96 & $0.60,1.52$ & 0.854 & 0.97 & $0.65,1.44$ & 0.875 \\
\hline \multicolumn{7}{|c|}{ ‘Sweet tooth’ pattern† } \\
\hline Q1 & 1 & & & 1 & & \\
\hline Q2 & 1.48 & $0.95,2.29$ & 0.080 & $1 \cdot 24$ & $0.82,1.87$ & 0.302 \\
\hline Q3 & 1.47 & $0.92,2.34$ & 0.105 & $2 \cdot 22$ & $1 \cdot 45,3.40$ & $<0.001$ \\
\hline Q4 & $2 \cdot 34$ & $1.47,3.73$ & 0.000 & 2.02 & $1.31,3.13$ & 0.002 \\
\hline \multicolumn{7}{|c|}{ 'Healthy' pattern† } \\
\hline Q1 & 1 & & & 1 & & \\
\hline Q2 & 0.71 & $0.48,1.05$ & 0.083 & 0.57 & $0.40,0.80$ & 0.001 \\
\hline Q3 & 0.37 & $0.23,0.60$ & $<0.001$ & 0.68 & $0.48,0.97$ & 0.034 \\
\hline Q4 & 0.50 & $0.31,0.79$ & 0.004 & 0.51 & $0.34,0.75$ & 0.001 \\
\hline \multicolumn{7}{|l|}{ Region } \\
\hline South & 1 & & & 1 & & \\
\hline North & 0.77 & $0 \cdot 49,1 \cdot 21$ & 0.261 & 2.49 & $1 \cdot 80,3.43$ & $<0.001$ \\
\hline
\end{tabular}

and anaemia. A validated food-frequency questionnaire was used in the study (Zhao et al. 2002). The limitation of the study is the potential confounders of anaemia that were not included in the study, for example infectious disease, environmental pollution and medication history. The prevalence of intestinal worm infestation is supposedly low ( $\mathrm{Xu}$ et al. 2004). In China, anaemia can be caused by iron deficiency or thalassaemia, the latter being quite common in southern parts of China (Wang et al. 1987; Cai et al. 2002; Xu et al. 2004). Because of the limitations of the study, we do not have data on serum Fe level, which would be necessary in order to distinguish between these two causes of anaemia. In our research area, the prevalence is, however, low (Zeng \& Huang, 1987).

Another limitation is the common one for factor analysis that has already been discussed $(\mathrm{Hu}, 2002$; Newby \& Tucker, 2004), for example that the results are strongly affected by subjective analytic but important decisions, such as the number of factors to extract, the variance explained. As we used more than one member of each household, there could be some bias in the association between diet and anaemia owing to the common dietary habit of the household. The sizes of these families were, however, so similar (combination of (1) man and woman; (2) man or woman with one child) that we do not expect a bias, because the representation from each household is the same, namely two.

The daily intakes of cereals and vegetables are relatively high in China, on average $402 \mathrm{~g}$ and $276 \mathrm{~g}$, respectively (Wang, 2005). The 'traditional' food pattern, characterised by a high intake of rice and vegetables, was positively associated with anaemia in our study. Men from first quartile of this pattern had the lowest prevalence of anaemia (7.7\%), compared with $24.8 \%$ in the fourth quartile group. The same trend was observed among women. This is consistent with present knowledge. Rice and vegetable contain non-haem Fe. The bioavailability of non-haem $\mathrm{Fe}$ in these foods is low 
(Hunt, 2002; Lopez \& Martos, 2004). The fourth quartile of the 'traditional' food group was characterised by a high intake of rice (average intake $467 \mathrm{~g} / \mathrm{d}$ ), whereas the first quartile of the 'traditional' pattern had an intake of about $377 \mathrm{~g} / \mathrm{d}$ of wheat flour. These represented two distinct staple patterns, based on either rice or wheat flour, which can be related to the regional differences between the north and south, as illustrated in Table 3. As the content of Fe in wheat flour is about twice as that in rice (Yang, 2005), this may also explain the difference in prevalence of anaemia according to the 'traditional' food pattern.

Factor 2 was characterised by a high consumption of meat and alcohol. Meat has an important role in Fe balance, not only because of its haem $\mathrm{Fe}$ content, but also because it enhances non-haem Fe absorption (Hallberg \& Rossander, 1984; Heath \& Fairweather-Tait, 2002). There was a trend of a negative, albeit not significant, association between 'macho' food and anaemia among men. Those from the first quartile of 'macho' food had the highest prevalence of anaemia $(23.6 \%)$, compared with $16.9 \%$ in the fourth quartile group. There was, however, no such association in women. This result was inconsistent with present knowledge about the association between meat, alcohol consumption and anaemia. The lack of such an association in our study may be attributable to the moderate consumption of meat in this population (mean daily intake of animal foods $59.9 \mathrm{~g}$ ). In the Chinese population, the average intake of animal foods and fish is only $78.6 \mathrm{~g}$ (Wang, 2005). It is also possible that this food pattern, which contains foods such as egg and soyabean products (tofu) will inhibit the absorption of non-haem Fe (Lopez \& Martos, 2004).

The 'sweet tooth' food pattern was found to be positively associated with anaemia. The prevalence of anaemia was higher for the highest quartile of the 'sweet tooth' food pattern compared with the lowest quartile (men: $21.5 \%$ v. $12.6 \%$; women: $34.1 \%$ v. $21.5 \%$ ). This food pattern is characterised by drinks, milk and cake. This finding is consistent with present knowledge. $\mathrm{Ca}$ and dairy products have been reported to have an inhibitory effect on non-haem absorption (Hallberg et al. 1991; Lopez \& Martos, 2004). Tea has also been found to be a strong inhibitor of Fe absorption (Zijp et al. 2000; Nelson \& Poulter, 2004). Although we did not have information on tea consumption in this study, the high consumption of drinks should theoretically be correlated with tea consumption because, in the food-frequency questionnaire interview, we defined tea as one of the components of drinks.

The highest quartile of the 'healthy food' pattern contained a large amount of vegetables and fruits, meeting the WHO recommendations (World Health Organization/Food and Agriculture Organization, 2003). At the same time, it also contained moderate amount of animal foods, some milk and whole grains. The risk of anaemia for people in the highest quartile of the 'healthy' food pattern was about $50 \%$ lower than that in people from the lowest quartile. The inverse association between the 'healthy' food pattern and anaemia may partly be explained by the fact that this pattern contained fruit. Ascorbic acid in fruit may play a role as ascorbic acid has been reported to enhance non-haem Fe absorption (Lopez \& Martos, 2004). Another factor may be that those having a 'healthy' food pattern may be more conscious about their health. As the food items included are cheap, these foods are recommended by the Chinese Nutrition Association. The 'healthy' food pattern might also be a proxy of other lifestyle factors. Studies from other countries show that healthy food patterns are associated with a healthy lifestyle (Park et al. 2005). Lifestyle factors other than diet may be involved, such as seeking out health services.

In the present study, SES was found to be associated with the four food patterns. High-SES participants had a high intake of 'traditional', 'macho' and 'sweet tooth' foods. People from low-SES categories seemed to have a more healthy food choice: a high proportion of both a high intake of the 'healthy' pattern and a low intake of the 'sweet tooth' pattern. The risk of anaemia was lower in the low-SES group than the high-SES group.

The association between SES and anaemia in multivariate analysis among women may suggest that other factors in addition to the identified food patterns play a role in the epidemic of anaemia. It might be that the high-SES women use more refined foods containing less $\mathrm{Fe}$. Although women from the north consumed more from the 'healthy' pattern and less from the 'traditional' and 'sweet tooth' patterns, in multivariate analysis adjusting for food patterns, women in the north had a $2 \cdot 5$-foldhigher risk of being anaemic than their counterparts in the south. This could be explained by the big economic difference between the south and the north. The gross domestic product of the north is only one third that of the south. The economic disparity may imply differences in the quality of housing, other environmental factors and health services between the two regions.

As the four food patterns include foods that both inhibit and enhance Fe absorption, the interactions between food components may play different roles in Fe absorption and give rise to different effects. The present study provides an insight into how food patterns are associated with anaemia in the Chinese context, although inferences about causal relationships cannot be made. Our results support the idea that choosing the right foods is important in the prevention of anaemia. Residence is an important factor that should be taken into account in intervening with anaemia. In the study area, anaemia intervention strategies could be different between the south and the north; for example, Fe-fortified wheat flour might be relevant in the north where it is the main staple foodstuff, but not in the south, where rice is dominant. Education on promoting healthy eating is encouraged in the prevention of anaemia.

\section{Acknowledgements}

The authors thank the participating Regional Centers for Disease Control and Prevention in Jiangsu province, including the Nanjing, Xuzhou, Jiangyin, Taicang, Suining, Jurong, Sihong, and Haimen Centres for their support for the data collection. The study is financed by Jiangsu Provincial Health Bureau.

\section{References}

ACC/SCN (Administrative Committee on Coordination SubCommittee on Nutrition) (2000) Fourth Report on the World Nutrition Situation. Geneva: ACC/SCN/International Food Policy Research Institute. 
Boccio JR \& Iyengar V (2003) Iron deficiency: causes, consequences, and strategies to overcome this nutritional problem. Biol Trace Elem Res 94, 1-32.

Cai R, Liu J, Wang L, Liang X, Xiao B, Su L, Zhou Y \& Pan L (2002) Prevalence survey and molecular characterization of alpha and beta thalassemia in Liuzhou city of Guangxi. Zhonghua Liu Xing Bing Xue Za Zhi 23, 281-285.

Chen J, Zhao X, Zhang X, et al. (2005) Studies on the effectiveness of NaFeEDTA-fortified soy sauce in controlling iron deficiency: a population-based intervention trial. Food Nutr Bull 26, 177-186; discussion 187-179.

Dallman PR (1984) Diagnosis of anemia and iron deficiency: analytic and biological variations of laboratory tests. Am J Clin Nutr 39, 937-941.

Hallberg L, Brune M, Erlandsson M, Sandberg AS \& Rossander-Hulten L (1991) Calcium: effect of different amounts on nonheme- and heme-iron absorption in humans. Am J Clin Nutr 53, 112-119.

Hallberg L \& Rossander L (1984) Improvement of iron nutrition in developing countries: comparison of adding meat, soy protein, ascorbic acid, citric acid, and ferrous sulphate on iron absorption from a simple Latin American-type of meal. Am J Clin Nutr 39, 577-583.

Harvey PW, Dexter PB \& Darnton-Hill I (2000) The impact of consuming iron from non-food sources on iron status in developing countries. Public Health Nutr 3, 375-383.

Heath AL \& Fairweather-Tait SJ (2002) Clinical implications of changes in the modern diet: iron intake, absorption and status. Best Pract Res Clin Haematol 15, 225-241.

Hu FB (2002) Dietary pattern analysis: a new direction in nutritional epidemiology. Curr Opin Lipidol 13, 3-9.

Hunt JR (2002) Moving toward a plant-based diet: are iron and zinc at risk? Nutr Rev 60, 127-134.

Jacques PF \& Tucker KL (2001) Are dietary patterns useful for understanding the role of diet in chronic disease? Am J Clin Nutr 73, $1-2$.

Jiangsu Bureau of Statistics (2002) Statistical Yearbook of Jiangsu. Beijing: China Statistics Press.

Kant AK (2004) Dietary patterns and health outcomes. J Am Diet Assoc 104, 615-635.

Kim JO \& Mueller CW (1978) Factor Analysis: Statistical Methods and Practical Issues. Thousand Oaks, CA: Sage.

Li L, Rao K, Kong L, Yao C, Xiang H, Zhai F, Ma G \& Yang X (2005) A description on the Chinese national nutrition and health survey in 2002. Chinese J Epidemiol 26, 474-484.
Lopez MA \& Martos FC (2004) Iron availability: An updated review. Int J Food Sci Nutr 55, 597-606.

Nelson M \& Poulter J (2004) Impact of tea drinking on iron status in the UK: a review. J Hum Nutr Diet 17, 43-54.

Newby PK \& Tucker KL (2004) Empirically derived eating patterns using factor or cluster analysis: a review. Nutr Rev 62, 177-203.

Park SY, Murphy SP, Wilkens LR, Yamamoto JF, Sharma S, Hankin JH, Henderson BE \& Kolonel LN (2005) Dietary patterns using the Food Guide Pyramid groups are associated with sociodemographic and lifestyle factors: the multiethnic cohort study. J Nutr 135, 843-849.

Semba RD \& Bloem MW (2002) The anemia of vitamin A deficiency: epidemiology and pathogenesis. Eur J Clin Nutr 56, 271-281.

Togo P, Osler M, Sorensen TI \& Heitmann BL (2001) Food intake patterns and body mass index in observational studies. Int $J$ Obes Relat Metab Disord 25, 1741-1751.

Wang L (2005) Report of Chinese National Nutrition and Health Survey in 2002. Beijing: People's Medical Publishing House.

Wang SW, Wang LM, Zhang BH, Wu GY, Huang YW, Wang RX \& Zhang NJ (1987) A survey of Hb Bart in cord blood and the alphaglobin gene in South China. Birth Defects Orig Artic Ser 23, $23-30$.

World Health Organization (2001) Iron Deficiency Anaemia. Assessment, Prevention and Control: A Guide for Programme Managers: WHO/NHD/01.3. Geneva: World Health Organization.

World Health Organization /Food and Agriculture Organization (2003) Diet, Nutrition and the Prevention of Chronic Diseases: Report of a Joint WHO/FAO Expert Consultation. Geneva: World Health Organization.

Xu XM, Zhou YQ, Luo GX, et al. (2004) The prevalence and spectrum of alpha and beta thalassaemia in Guangdong Province: implications for the future health burden and population screening. J Clin Pathol 57, 517-522.

Yang Y (2005) Chinese Food Composition Table 2004. Beijing: Peking University Medical Press.

Zeng YT \& Huang SZ (1987) Disorders of haemoglobin in China. J Med Genet 24, 578-583.

Zhao W, Hasegawa K \& Chen J (2002) The use of food-frequency questionnaires for various purposes in China. Public Health Nutr 5, 829-833.

Zijp IM, Korver O \& Tijburg LB (2000) Effect of tea and other dietary factors on iron absorption. Crit Rev Food Sci Nutr 40, $371-398$. 